\title{
Identification of GRP75 as an Independent Favorable Prognostic Marker of Neuroblastoma by a Proteomics Analysis
}

\author{
Wen-Ming Hsu, ${ }^{1}$ Hsinyu Lee, ${ }^{4,5}$ Hsueh-Fen Juan, ${ }^{4,6}$ Yu-Yin Shih, ${ }^{4,7}$ Bo-Jeng Wang, ${ }^{7}$ Chien-Yuan Pan, ${ }^{4,5}$ \\ Yung-Ming Jeng, ${ }^{2}$ Hsiu-Hao Chang ${ }^{3}$ Meng-Yao Lu, ${ }^{3}$ Kai-Hsin Lin, ${ }^{3}$ Hong-Shiee Lai, ${ }^{1}$ \\ Wei-Jao Chen, ${ }^{1}$ Yeou-Guang Tsay, ${ }^{8}$ Yung-Feng Liao, ${ }^{5,7}$ and Fon-Jou Hsieh ${ }^{9}$
}

\begin{abstract}
Purpose: Neuroblastoma (NB) is a heterogeneous neoplasm. Detailed biological discrimination is critical for the effective treatment of this disease. Because the tumor behavior of NB is closely associated with the histologic state of differentiation, we thus aimed to identify novel differentiation-associated markers of NB with prognostic implication.

Experimental Design: A human NB cell line SH-SY5Y was used as a model system to explore potential biomarkers for the differentiation of NB by proteomic analyses. Seventy-two NB tumor tissues were subsequently investigated by immunohistochemistry to validate the correlations between the expression of a novel prognostic marker, various clinicopathologic and biological factors, and patient survival.

Results: Using two-dimensional differential gel electrophoresis, we found a total of 24 spots of proteins in SH-SY5Y cells whose expression was enhanced following differentiation. Glucoseregulated protein 75 (GRP75) was unambiguously identified as one of the five proteins that were dramatically up-regulated following differentiation. Immunohistochemical analyses of $72 \mathrm{NB}$ tumor tissues further revealed that positive GRP75 immunostaining is strongly correlated with differentiated histologies $(P<0.001)$, mass-screened tumors $(P=0.016)$, and early clinical stages $(P<$ $0.001)$ but inversely correlated with MYCN amplification $(P=0.010)$. Univariate and multivariate survival analyses showed that GRP75 expression is an independent favorable prognostic factor. Conclusions: The present findings clearly showed that our proteomics-based novel experimental paradigm could be a powerful tool to uncover novel biomarkers associated with the differentiation of NB. Our data also substantiate an essential role of GRP75 in the differentiation of NB.
\end{abstract}

Neuroblastoma (NB) is the most common extracranial solid tumor of infancy and childhood with an incidence of 8.0 per million per year (1), and this tumor arises from primitive neuroepithelial cells of the neural crest and occurs most

Authors' Affiliations: Departments of ${ }^{1}$ Surgery, ${ }^{2}$ Pathology, and ${ }^{3}$ Pediatrics, National Taiwan University Hospital and National Taiwan University College of Medicine; ${ }^{4}$ Department of Life Science and Institutes of ${ }^{5}$ Zoology and ${ }^{6}$ Molecular and Cellular Biology, National Taiwan University; ${ }^{7}$ Institute of Cellular and Organismic Biology, Academia Sinica; ${ }^{8}$ Institute of Biochemistry and Molecular Biology and Proteomics Research Center, National Yang-Ming University; and ${ }^{9}$ Graduate Institute of Clinical Medicine, National Taiwan University College of Medicine, Taipei, Taiwan

Received 9/12/07; revised 3/18/08; accepted 5/2/08

Grant support: National Science Council of Taiwan grants NSC 94-2314-B-002 287 (W-M. Hsu) and NSC 95-2320-B010-054-MY2 (Y-G. Tsay), National Taiwan University Hospital grant NTUH 95-000351 (W-M. Hsu), National Yang-Ming University (Y-G. Tsay), and Academia Sinica (Y-F. Liao)

The costs of publication of this article were defrayed in part by the payment of page charges. This article must therefore be hereby marked advertisement in accordance with 18 U.S.C. Section 1734 solely to indicate this fact.

Note: Supplementary data for this article are available at Clinical Cancer Research Online (http://clincancerres.aacrjournals.org/).

Y.-G. Tsay, Y.-F. Lao, and F.-J. Hsieh contributed equally to this work.

Requests for reprints: Fon-Jou Hsieh, Graduate Institute of Clinical Medicine, National Taiwan University College of Medicine, 7 Chung-Shan South Road, Taipei 100, Taiwan. Phone: 886-2-23562614; Fax: 886-2-23934197; E-mail: fjhsieh@ntu.edu.tw.

(C) 2008 American Association for Cancer Research

doi:10.1158/1078-0432.CCR-07-4181 frequently in the adrenal gland (2). NB is quite a heterogeneous tumor and presents a broad clinical and biological spectrum ranging from highly undifferentiated tumors with very poor outcomes to the most differentiated benign ganglioneuroma or NB with high probability of spontaneous regression and hence favorable prognosis. At least two categories of NB have been identified $(3,4)$. One, the favorable NB, is associated with young age and early stage at diagnosis, triploid karyotypes with whole chromosome gains, and excellent clinical outcome despite minimal or no therapy. The other, the unfavorable $\mathrm{NB}$, is associated with older age and advanced stage and pseudodiploid karyotypes with structural changes including deletion of $1 \mathrm{p}$ or $11 \mathrm{q}$, unbalanced gain of $17 \mathrm{q}$, and/or amplification of the MYCN proto-oncogene. Patients with unfavorable NB usually have a very poor outcome despite multimodality therapies including bone marrow transplantation. Furthermore, we have also shown previously that the biological characteristics of NB tumors would significantly influence the surgical decision (5). We showed that gross total resection, a highly risky operation and controversial treatment for $\mathrm{NB}$, of the primary tumor could carry favorable outcome only in a specific group of NB with certain biomarkers (5). These lines of evidence suggest that a more detailed understanding of the clinical and biological characteristics of $\mathrm{NB}$ is imperative for the selection of appropriate therapeutic intervention on these tumors to achieve effective treatment without unnecessary complications. 


\section{Translational Relevance}

The present findings clearly showed that our proteomics-based novel experimental paradigm could be a powerful tool to uncover novel biomarkers associated with the differentiation of neuroblastoma. Our present study shows the establishment and application of a novel proteomics-based experimental paradigm for the identification of NB tumor biomarkers. Evaluation of GRP75 expression in tumor tissues of NB may provide complementary prognostic information for further subclassification of these tumors, which in turn may help to determine the most appropriate strategy of treatment. Furthermore, because the expression of GRP75 strongly correlates with the differentiation of NB, GRP75 allows itself as a potential target of the treatment of NB. Together, the present data delineate a good example for the employment of proteomic approaches in the identification of potential biomarkers of cancers and recognize the potential of GRP75 as a therapeutic target of NB.

Although the pathogenesis of NB remains obscure, it has been suggested that aberrant regulation of cellular differentiation could contribute to the development of this cancer (6). Coincided with this concept, the prognosis of NB patients has been shown to be closely associated with the histologic grade of differentiation in tumors from NB patients (7). Most biomarkers for favorable outcome in NB, such as HNK-1, Trk-A, $\mathrm{H}$-Ras, glucose-regulated protein 78 (GRP78), and calreticulin, are associated with the differentiation of NB cells $(8-12)$. Here, we employ a proteomic approach to look for novel biomarkers for cellular differentiation that could play a pivotal role in the pathogenesis of NB. Recent advances in proteomic technologies have allowed the identification of proteins whose amounts and states could be altered by the disease process in a high-throughput manner, making proteomic analysis one of the most valuable tools for defining biomarkers of various tumors $(13,14)$.

The present study successfully identified GRP75 (74 kDa; $\mathrm{pI}$ 5.6) as a novel biomarker of NB. GRP75 is a member of heat shock protein 70 family and is first cloned from the cytoplasmic fraction of normal mouse fibroblast (15). In addition to its primary residence in mitochondria, GRP75 is also present at endoplasmic reticulum, plasma membrane, cytoplasmic vesicles, and cytosol (16). It involves in cellular functions ranging from stress response, intracellular trafficking, antigen processing, control of cell proliferation, differentiation, and tumorigenesis (17). Our data have confirmed that the expression of GRP75 in NB tumor tissues strongly correlates with differentiated histologies and predicts a favorable patient's outcome. GRP75 could thus play a critical role in the differentiation of NB.

\section{Materials and Methods}

Experimental procedures for cell culture and treatment, protein extraction and Cy dye labeling, two-dimensional differential gel electrophoresis (DIGE) and imaging analysis, liquid chromatography- tandem mass spectrometry analysis, and transfection of a small interfering RNA targeting GRP75 are included in the Supplementary Data.

\section{Western blot analysis}

Two-dimensional Western blot. To show the specifically enhanced expression of GRP75 after the differentiation of NB cells, proteins extracted from SH-SY5Y cells treated with DMSO $(0.1 \%)$ or all-trans retinoic acid (ATRA; $10 \mu \mathrm{mol} / \mathrm{L}$ ) for 5 days were resolved by twodimensional electrophoresis. Proteins were blotted onto a nitrocellulose membrane electrophoretically and incubated with a goat anti-GRP75 antibody (1:200; Santa Cruz Biotechnology). The immunoreactive spots were visualized with ECL Plus Western Blotting Detection System (Amersham Biosciences)

One-dimensional Western blot. The details of protein extraction from cells or tumor tissues, electrophoresis, and immunoblotting have been described previously $(11,18)$. The antibodies used and their dilution were as follows: goat anti-GRP75 antibody (1:200; Santa Cruz Biotechnology), rabbit anti-glyceraldehyde-3-phosphate dehyhrogenase antibody $(1: 1,000$; Santa Cruz Biotechnology), mouse anti-neuronspecific enolase (1:1,000; Millipore), and horseradish peroxidaseconjugated secondary antibodies (1:5,000; Santa Cruz Biotechnology). The expression levels of GRP75, calreticulin, and neuron-specific enolase were assessed by densitometry and normalized with those of glyceraldehyde-3-phosphate dehyhrogenase, the internal control. Data were shown as the mean $\pm \mathrm{SD}$ of the averages of triplicate measurements from three independent experiments.

Indirect immunofluorescence staining and confocal microscopy SH-SY5Y cells were grown on coverslips and incubated in DMEM/ Ham's F-12 containing $2 \%$ fetal bovine serum followed by the treatment with DMSO $(0.1 \%)$ or ATRA $(10 \mu \mathrm{mol} / \mathrm{L})$ for 5 days. Cells were then fixed by $4 \%$ paraformaldehyde in PBS at room temperature for $60 \mathrm{~min}$ and permeabilized by $10 \%$ formaldehyde in PBS containing $0.1 \%$ Triton $\mathrm{X}-100$ at room temperature for $10 \mathrm{~min}$. Detergent-permeabilized cells were blocked with $\mathrm{PBS} / 2 \%$ bovine serum albumin at room temperature for at least $1 \mathrm{~h}$ and were incubated with a goat anti-GRP75 antibody in PBS/0.1\% bovine serum albumin at $4{ }^{\circ} \mathrm{C}$ overnight. Following extensive washes with PBS, cells were incubated with an Alexa Fluor 488 donkey anti-goat IgG in PBS/0.1\% bovine serum albumin at room temperature for $1 \mathrm{~h}$. Following the removal of unbound antibodies by washes with PBS, the costaining of actin was done by repeating the staining procedure using a mouse anti-actin antibody as the primary antibody and an Alexa Fluor 647 goat anti-mouse IgG as the secondary antibody in the procedure described above. Nuclei were visualized by 4',6-diamidino2-phenylindole. Fluorophore-conjugated antibodies and 4',6-diamidino-2-phenylindole were obtained from Invitrogen. Immunofluorescence images were obtained by using a Leica TCS-SP5 laser scanning confocal microscope. The fluorescence intensity of GRP75 was quantitated by Leica Application Suite Advanced Fluorescence and analyzed by Student's $t$ test. Data were shown as the mean \pm SD of the average fluorescence per cell from five different viewing areas. The numbers of cells that were subject to fluorescence quantitation were 279 for DMSO-treated cells and 413 for ATRA-treated ones, respectively.

\section{ATRA-induced neuronal differentiation of NB cells}

SH-SY5Y human NB cells that were transfected with a GRP75-specific small interfering RNA or a nonspecific one (Mock) were then treated with $10 \mu \mathrm{mol} / \mathrm{L}$ ATRA or vehicle alone (0.1\% DMSO) in DMEM/Ham's F-12 containing $10 \%$ fetal bovine serum followed by incubation at $37^{\circ} \mathrm{C}$ for 3 days. Treated SH-SY5Y cells were harvested and lysed by PLB (Promega) containing the Complete protease inhibitor cocktail. Clarified lysates containing equivalent amounts of proteins were resolved by SDS-PAGE. The expression levels of calreticulin and neuron-specific enolase, two markers for the neuronal differentiation 
of NB cells $(12,19)$, were visualized by Western blotting using specific antibodies. The level of glyceraldehyde-3-phosphate dehyhrogenase was also determined as a protein load control.

To quantify neurite outgrowth of NB cells induced by ATRA, 150 or more transfected SH-SY5Y cells, whose dendritic trees were relatively isolated and did not have discontinuities in their dendritic trees, were chosen for quantification of neurite length. Image for Windows was the morphometric program used to measure the neurite length.

\section{Patients and treatment}

In a period of 14 years (from December 1990 to December 2004), 72 histologically proven NB patients with complete follow-up were enrolled in this study. Eight of these 72 patients were diagnosed by mass screening of urinary vanillyl-mandelic acid without any symptoms. This group of patients carried a male-to-female ratio of 40:32 and a median age at diagnosis of 2.5 years (range, $0-11.5$ years). The majority of tumors (41 cases) were originated primarily from the adrenal gland and others from retroperitoneum (18 cases), mediastinum ( 6 cases), neck ( 4 cases), and pelvis ( 3 cases). Based on the criteria of the International Neuroblastoma Pathology Classification, the differentiating status of the tumor histology was categorized into undifferentiated NB, differentiating NB (including poorly differentiated subtype), and ganglioneuroblastoma (GNB), distinguishable by the percentage and degree of differentiation of the NB cells $(11,20,21)$. Both differentiating NB and GNB were pooled as differentiated NB for the survival analysis. The distinction of tumor staging was based on the International Neuroblastoma Staging System (22). MYCN status of the tumor tissue was evaluated by fluorescence in situ hybridization analysis of formalin-fixed, paraffin-embedded tissues or fresh tumor single cells $(23,24)$. Patients were treated with surgery only or a combination of multiple modalities including chemotherapy, radiotherapy, and/or autologous bone marrow transplantation according to the patient's risk grouping (25). The median follow-up after diagnosis was 48.0 months with a range of 1 to 168 months, and the overall 5 -year survival rate in this cohort was 53.9\%.

\section{Immunohistochemical staining}

To validate the expression of GRP75 in NB, 72 formalin-fixed, paraffin-embedded tumor tissues that were obtained before chemotherapy were evaluated by immunohistochemical staining with a standard streptavidin-biotin method (11). Briefly, the paraffin sections $(5 \mu \mathrm{m})$ of tumors were dewaxed, rehydrated, and incubated with a goat anti-GRP75 antibody $(1: 100)$ at $4{ }^{\circ} \mathrm{C}$ overnight. Antibody-reactive sections were allowed to interact with the $\mathrm{N}$-Histofine Simple Stain MAXPO (Nichirei) for $30 \mathrm{~min}$ at room temperature followed by color development using diaminobenzidine. The nuclei were counterstained with hematoxylin. One ganglioneuroma tumor with consistent GRP75 expression by immunohistochemistry was used as a positive control. Nonimmunized goat serum was used to rule out nonspecific immunostaining. Tumors with various differentiating histologies were included in each staining. The immmunoreactivity of GRP75 was assessed by one pathologist who was blinded to the clinical backgrounds of the patients. A specific blocking peptide containing the reactive epitope of the anti-GRP75 antibody was used to compete the binding of anti-GRP75 for the determination of its specificity.

\section{Statistical analysis}

SPSS 10.0 for Windows software was used to perform the statistical analyses. The significance of the variations between the data resulted from different treatments was analyzed by Student's $t$ test. Associations between pairs of categorical variables were assessed with Pearson's $\chi^{2}$ test. Survival probabilities in various subgroups were estimated using the Kaplan-Meier method and analyzed by log-rank tests. The influence of each variable on survival was assessed by the multivariate Cox proportional hazard model. All statistical tests were two-sided, and those with a $P \leq 0.05$ were considered to be statistically significant.

\section{Results}

\section{Identification of differentiation-associated proteins in NB cells} by two-dimensional DIGE

SH-SY5Y cells treated with ATRA for 5 days showed morphologic characteristics of differentiation with prominent neurite extension as well as increased expression of calreticulin and neuron-specific enolase (data not shown). The proteins extracted from untreated and differentiated cells were stained with $\mathrm{Cy} 3$ and $\mathrm{Cy} 5$, respectively, and analyzed by twodimensional DIGE. A total of 24 orange/red spots that indicate protein candidates with enhanced expression after differentiation were highlighted for the entire cellular proteome (Fig. 1A, arrowheads). The amounts of most proteomic constituents were not significantly altered in differentiated cells versus undifferentiated ones, suggesting that the increased expression of those protein candidates is a specific response to ATRA-induced
Fig. 1. $A$, differential two-dimensional proteomic analysis of NB cells before and after differentiation. Red spots, increased expression after cell differentiation; green spots, decreased expression after cell differentiation; yellow spots, unchanged expression after cell differentiation. $B$, area containing the spots 1 to 4 is enlarged to show the circular areas in undifferentiated cells (left) and differentiated cells (right) for assessment of the respective fluorescent signals. $C$, area containing the spots 5 is enlarged to show the circular areas in undifferentiated cells (left) and differentiated cells (right) for assessment of the respective fluorescent signal. D, GRP75 expression in undifferentiated and differentiated NB cells evaluated by two-dimensional Western blot.

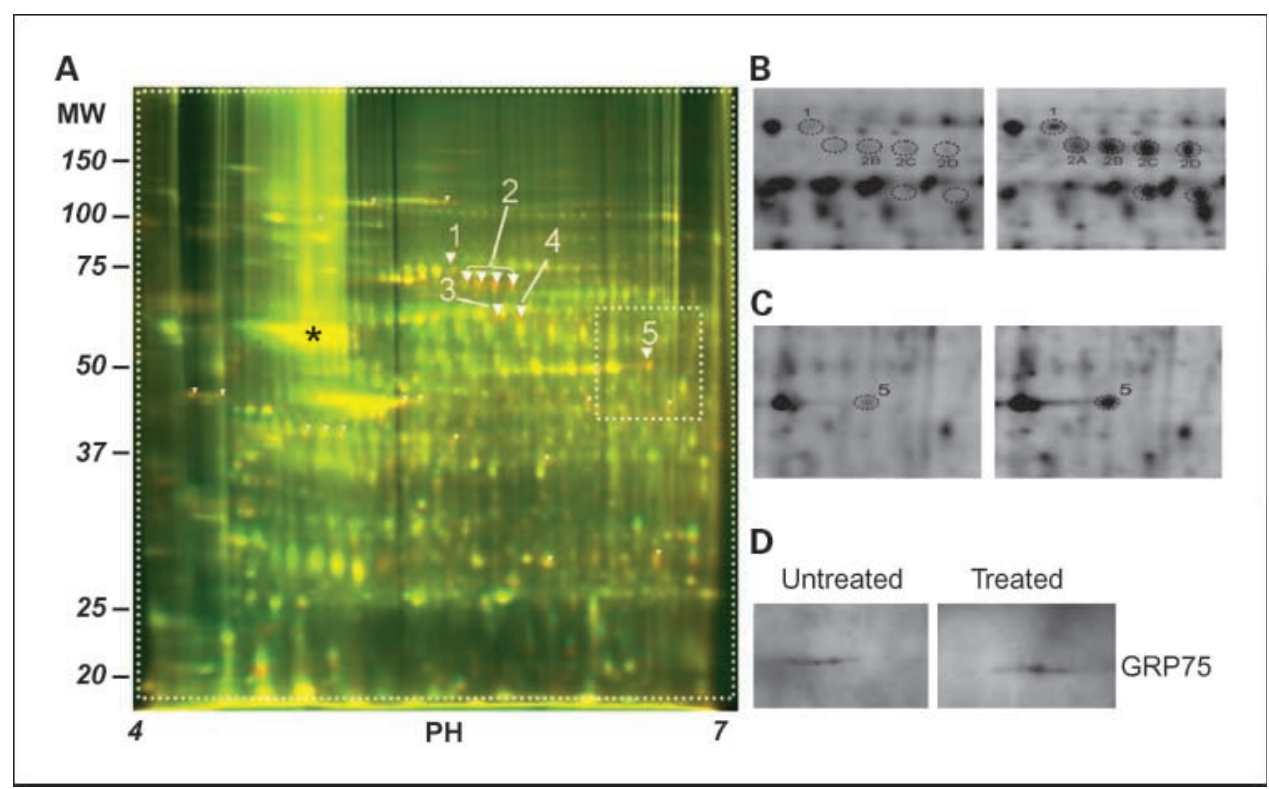




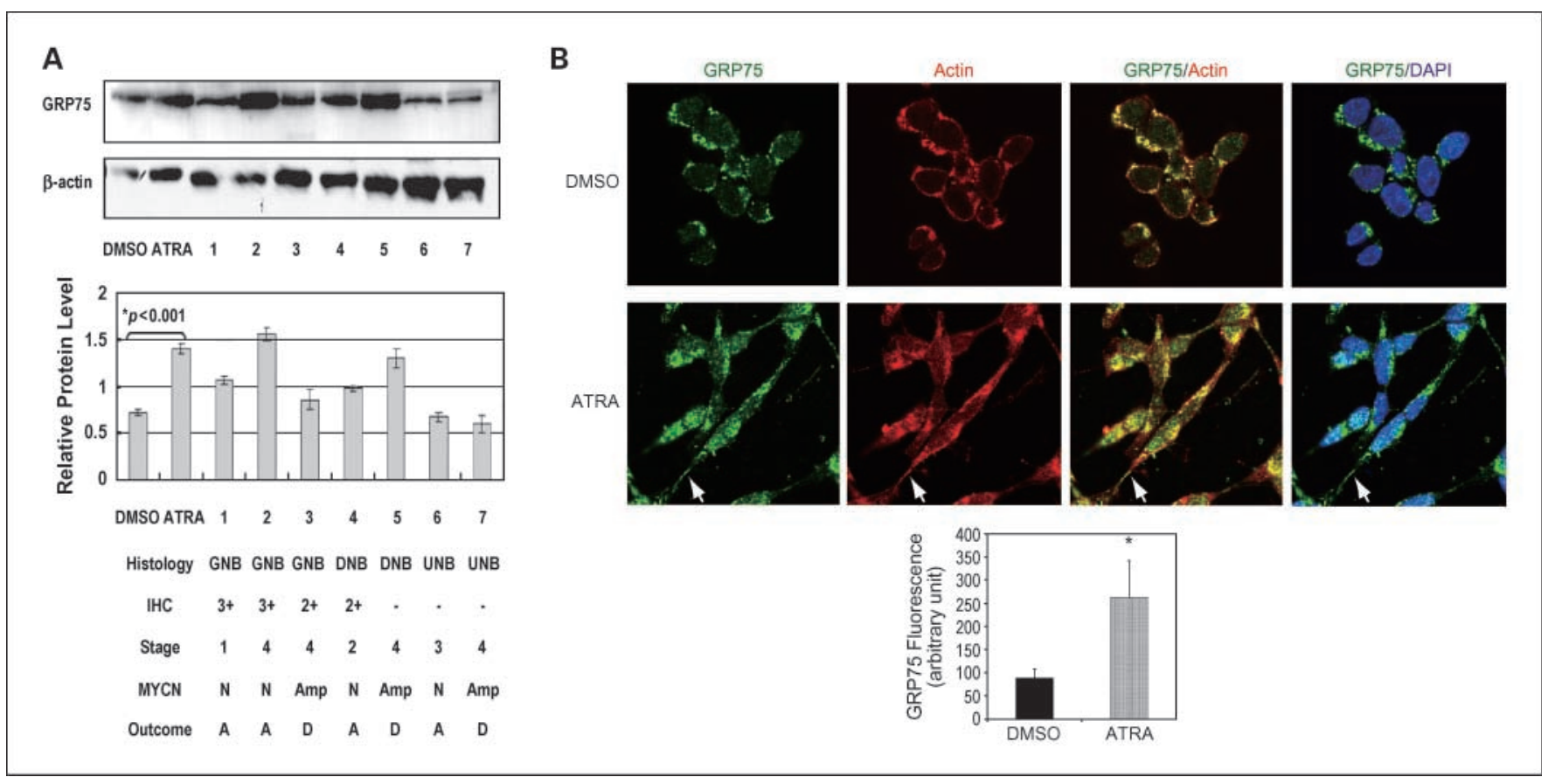

Fig. 2. A, GRP75 protein levels evaluated by Western blot in SH-SY5Y cells treated with DMSO or ATRA and in 7 NB tumors (cases 1-7). The histopathology, GRP75 immunohistochemical staining $(I H C)$ grading, clinical stage, MYCN status, and patient outcome of the NB tumors are depicted for comparison. $D N B$, differentiating NB; UNB, undifferentiated NB; $N$, nonamplified; $A m p$, amplified; $A$, alive; $D$, dead. $B$, immunolocalization and immunofluorescence intensity of GRP75 in undifferentiated and differentiated NB cells.

differentiation. Among these protein spots, eight of the most abundant ones were analyzed for the extent of the increases. The fluorescent signals of a protein spot in different proteomes were determined as signal percentages in respective proteomes (Supplementary Table S1). Using this approach, we estimated that the signal percentages of tubulin, one of the most intensely stained proteins (Fig. 1A, asterisk), remained largely unchanged, comprising $9.8 \%$ and $9.2 \%$ of the total signals in untreated and differentiated cells, respectively. We found that the fold of change for these up-regulated proteins during differentiation ranged from 1.7 to 2.7 (Fig. 1B and C; Supplementary Table $\mathrm{S} 1)$. The above protein spots were also subject to liquid chromatography-tandem mass spectrometry analyses to reveal their identities. Because four of the eight protein spots (Fig. 1A, spots 2A, 2B, 2C, and 2D) share similar results, these eight spots actually represented a total of five proteins, including GRP75 (spot 1), heat shock protein 2 (spots 2A, 2B, 2C, and $2 \mathrm{D}$ ), protein disulfide isomerase $\mathrm{A} 3$ precursor (spot 3 ), subunit $1 \beta$ of TCP1-containing chaperonin, (spot 4), and Eno1 protein (spot 5) (Supplementary Table S1). Our data also revealed that the expression of these proteins during differentiation was a moderate increase and not likely an all-or-none phenomenon. Among these identified proteins, GRP75 is a member of heat shock protein 70 family and has been suggested to play a functional role in the control of tumorigenesis (17). In addition, we have shown previously that GRP78, another member of the heat shock protein 70 family, is a favorable prognostic marker for NB (11). These findings prompted us to examine the differential expression and the function of GRP75 in $\mathrm{NB}$, validating the efficiency of proteomic approach in identifying key proteins involved in the differentiation of NB cells.

\section{Enhanced GRP75 expression in differentiated NB cells}

To confirm the specifically enhanced expression of GRP75 after differentiation, we performed two-dimensional Western blotting by using a specific anti-GRP75 antibody. In the lysates from both undifferentiated and differentiated cells, several protein spots of $75 \mathrm{kDa}$ and $\mathrm{pI} 5$ to 6 were found to be specifically reactive with this anti-GRP75 antibody (Fig. 1D). Our data also showed that the expression of GRP75 is significantly increased after cell differentiation, in accordance with the results obtained from two-dimensional DIGE analysis. Using one-dimensional Western blotting analysis, we further quantitatively showed that protein levels of GRP75 in ATRAtreated NB cells are increased approximately 1.8 -fold on differentiation (Fig. 2A, ATRA versus DMSO control, $P<$ $0.001, t$ test).

\section{Dramatic change in the subcellular distribution of GRP75 during the induced differentiation of NB cells}

Differential subcellular distribution of GRP75 that is concomitant with cellular immortalization has been observed in normal versus cancerous cells (16), prompting us to examine the subcellular localization of GRP75 in undifferentiated and differentiated NB cells. Using immunofluorescence staining, we found that ATRA-treated SH-SY5Y cells show a significant increase in the staining intensity of GRP75 (an average of 262 \pm 80 fluorescence unit per cell) with a pan-cytoplasmic distribution, whereas those treated with DMSO show a lower level of GRP75 (an average of $88 \pm 19$ fluorescence unit per cell) with a juxtanuclear pattern (Fig. 2B). In differentiated cells, the overall staining intensity of GRP75 became prominent, consistent with the notion that enhanced GRP75 expression correlates with the differentiation of NB. The neuritic localization, as 
defined by actin staining, of GRP75 can also be vividly observed in ATRA-treated cells (Fig. 2B, arrows), implicating a possible function of GRP75 in neurite formation.

\section{Correlation between the differential expression of GRP75 and the differentiation status of NB tumors}

The expression levels of GRP75 protein in seven NB tumor tissues, including three GNB, two differentiating NB, and two undifferentiated NB, were evaluated by Western blot analyses. Consistent with the data obtained with cultured NB cells, we found that tumors displaying better differentiation (GNB or differentiating NB) exhibit higher expression levels of GRP75 protein in general (Fig. 2A). However, there was a lack of clear statistical correlation between histologic grading and the protein expression of GRP75 evaluated by Western blotting. This discrepancy probably reflected the cellular heterogeneity in tumor tissues $(12,26)$. To circumvent this problem, we performed immunohistochemical staining of GRP75 in NB tumor tissues to assess the GRP75 expression in individual cells. We found that positive GRP75 staining could be specifically observed in ganglion cells of a ganglioneuroma tumor tissue and seems to display a pancytoplasmic distribution (Fig. 3A, 1, arrows), whereas the protein expression of GRP75 was negligible in Schwannian stromal cells. The specificity of the anti-GRP75 antibody for immunohistochemistry was confirmed by a competition study, which showed that the GRP75 immunostaining was blocked completely by the specific peptide (Fig. 3A, 2). In addition to the mature ganglion cells, neuroblastic cells with various differentiating states also showed positive GRP75 immunostaining in the cytoplasm (Fig. 3A, 4-6, arrows), whereas undifferentiated neuroblastic cells usually showed negative staining (Fig. 3A, 3). Immunohistochemistry thus indicates a more specific method to assess the GRP75 protein expression in the neuroblastic cells among a heterogeneous NB tumor.

GRP75 expression and clinicopathologic and biological factors

For further understanding the association between GRP75 protein expression and cellular differentiation as well as its clinical significance, we then extended our studies to a larger patient population using immunohistochemical evaluation of GRP75 expression in NB tumor samples. These NB tumors were classified based on their immunoreactivity of GRP75 into four categories: "-" (no expression, no stained cells or only isolated single stained cells seen), "1+" (weak expression, 10-35\% of neuroblastic cells stained), " $2+$ " (moderate expression, 35-70\% of neuroblastic cells stained), and " $3+$ " (strong expression, $>70 \%$ of neuroblastic cells stained; Fig. 3A, 3-6). Tumors were assigned into negative GRP75 protein expression (" - " in immunoreactivity) and positive GRP75 protein expression (" $1+$, , " $2+$, , or " $3+$ " in immunoreactivity) for the statistical analysis. We found that immunoreactivities $(1+$ to $3+)$ of GRP75 could be detected in 34 of the $72 \mathrm{NB}(47.2 \%)$. The intensity and percentage of positive GRP75 immunostaining

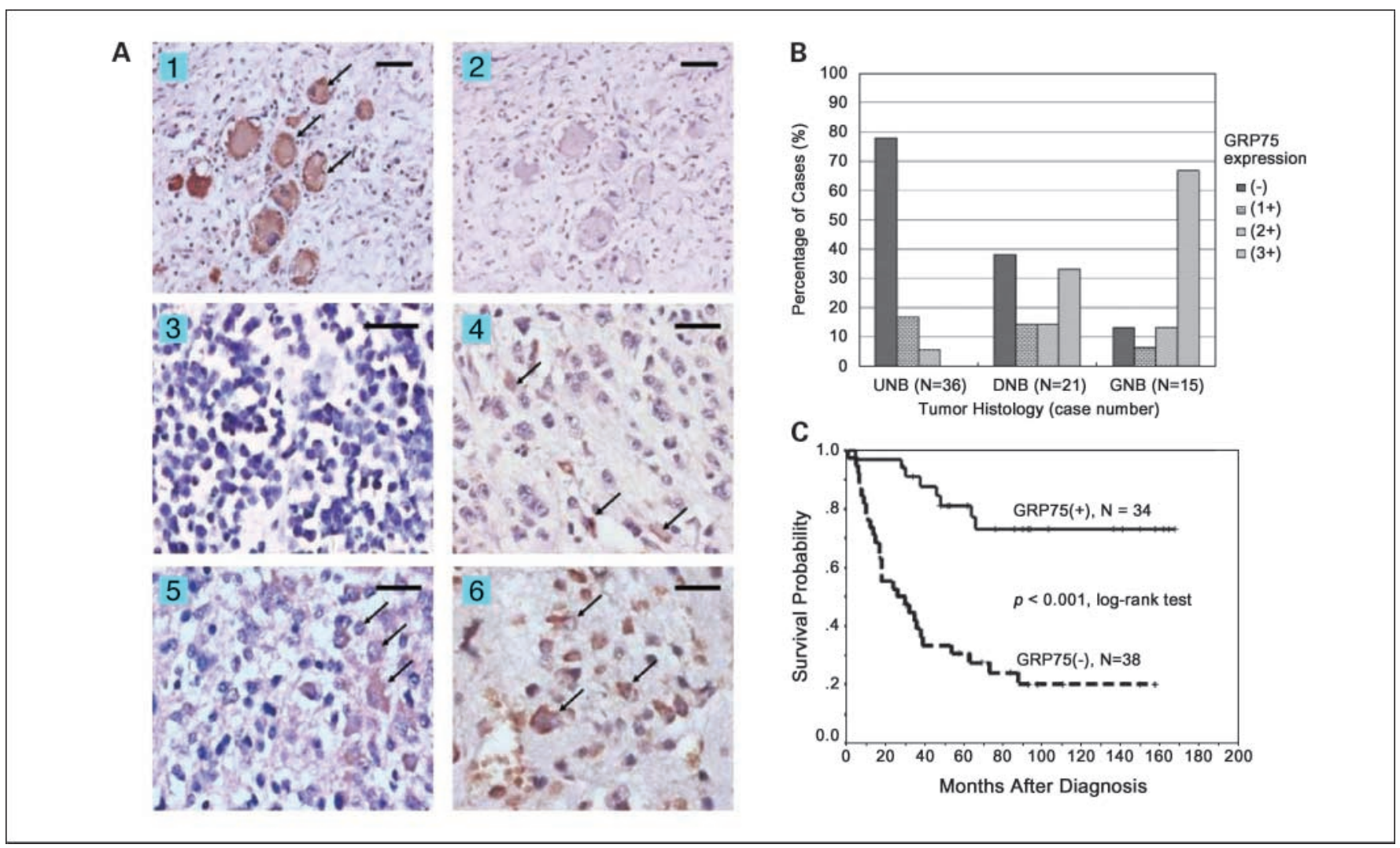

Fig. 3. Immunohistochemical analysis of GRP75 expression in NB. $A 1$, positive control GRP75 immunostaining of a ganglioneuroma; $A 2$, positive control GRP75 immunostaining blocked by the specific peptide; $A 3$, "-" GRP75 immunostaining; $A 4$, "1+" GRP75 immunostaining; $A 5$, "2+" GRP75 immunostaining; $A 6$, " $3+$ + GRP75 immunostaining. Bar, $100 \mu \mathrm{m}$. $B$, percentage distribution of GRP75 expression in undifferentiated NB, differentiating NB, and GNB. $C$, Kaplan-Meier survival analysis according to the expression of GRP75 in 72 NB patients. 
correlated strongly with the differentiation of tumor histology. As the tumor histology displayed a differentiation pattern, the intensity and percentage of positive GRP75 immunostaining increased simultaneously (Fig. 3B; $P<0.001$ ). The relationship between the GRP75 protein expression and the clinicopathologic and biological variables of $\mathrm{NB}$ was summarized in Table 1. In addition to histologic grade of differentiation, positive GRP75 immunostaining also correlated strongly with tumors detected by mass screening $(P=0.016)$ as well as early clinical stages (stages I, II, and IVS; $P<0.001$ ). Furthermore, there was an inverse correlation between GRP75 protein expression and the unfavorable biomarker of MYCN amplification $(P=0.010)$.

\section{Patients with positive scores for GRP75 expression showed favorable clinical outcomes}

Kaplan-Meier analysis showed that patients with positive GRP75 protein expression had a significantly better predictive 5 -year survival rate than patients with negative GRP75 protein expression (81.2\% and 30.7\% respectively: Fig. 3 C; $P<0.001$, log-rank test). Univariate analysis showed that, in addition to GRP75 expression, patient's age $\leq 1$ year, early clinical stages (stage I, II, or IVS), and differentiated histology (including differentiating $\mathrm{NB}$ and $\mathrm{GNB}$ ) also correlate with a better survival, whereas MYCN amplification predicts a very poor outcome (Table 2). Multivariate analysis by the Cox proportional hazard model showed that, in addition to clinical stage and MYCN status, GRP75 expression is also an independent prognostic factor (Table 2).

To understand the significance of GRP75 expression in the prognostic discrimination, the effect of GRP75 protein expression on patient survival was further analyzed against tumor histology, clinical stage, and MYCN status. Positive GRP75 expression clearly predicted a better survival in patients with either undifferentiated or differentiated histologies of $\mathrm{NB}$ (Fig. 4A and $\mathrm{B} ; P=0.002$ and 0.025 , respectively). Because most patients with early-stage NB (stages I, II, and IVS) showed positive GRP75 protein expression with favorable outcomes, they could not be discriminated by GRP75 expression for their prognosis. Nevertheless, in patients with advanced-stage diseases (stage III or IV), the prognosis could be clearly distinguished by GRP75 protein expression (Fig. 4C; $P=0.005)$. Although 7 of 15 patients (46.7\%) died of their disease in the category of advanced stages and positive GRP75 protein expression, 28 of 33 patients $(84.8 \%)$ died in the category of advanced stages and negative GRP75 expression. The prognosis of NB patients with MYCN amplification could not be distinguished by GRP75 expression due to the low frequency of positive GRP75 protein expression and very poor outcome for these patients. For the patients without MYCN amplification, positive GRP75 protein expression clearly predicted a favorable outcome (Fig. 4D; $P<0.001$ ). Whereas only 6 of $31(19.4 \%)$ patients with normal MYCN copy number and positive GRP75 protein expression died of their disease, 16 of 25 (64\%) patients with normal MYCN copy number and negative GRP75 protein expression died of their disease. Together, our data clearly showed that altered GRP75 expression is a signature of differentiated NB tumors.

\section{Discussion}

Our present study shows the establishment and application of a novel proteomics-based experimental paradigm for the

Table 1. GRP75 expression and clinicopathologic and biological characteristics of NB

\begin{tabular}{|c|c|c|c|}
\hline Variable & Cases & Positive GRP75 expression (\%) & $\boldsymbol{P} *$ \\
\hline \multicolumn{4}{|l|}{ Mass screening } \\
\hline Yes & 8 & $7(87.5)$ & \multirow[t]{2}{*}{0.016} \\
\hline No & 64 & $27(42.2)$ & \\
\hline \multicolumn{4}{|l|}{ Sex } \\
\hline Male & 40 & $20(50.0)$ & \multirow[t]{2}{*}{0.598} \\
\hline Female & 32 & $14(43.8)$ & \\
\hline \multicolumn{4}{|l|}{ Age at diagnosis (y) } \\
\hline$\leq 1$ & 22 & $14(63.6)$ & \multirow[t]{2}{*}{0.064} \\
\hline$>1$ & 50 & $20(40.0)$ & \\
\hline \multicolumn{4}{|l|}{ Clinical stage } \\
\hline I & 6 & $5(83.3)$ & \multirow[t]{5}{*}{$<0.001^{\dagger}$} \\
\hline II & 13 & $10(76.9)$ & \\
\hline IVS & 5 & $4(80.0)$ & \\
\hline III & 10 & $2(20.0)$ & \\
\hline IV & 38 & $13(34.2)$ & \\
\hline \multicolumn{4}{|l|}{ Primary tumor site } \\
\hline Adrenal & 41 & $19(46.3)$ & \multirow[t]{2}{*}{0.863} \\
\hline Extra-adrenal & 31 & $15(48.4)$ & \\
\hline \multicolumn{4}{|l|}{ Tumor histology } \\
\hline Undifferentiated NB & 36 & $8(22.2)$ & \multirow[t]{3}{*}{$<0.001$} \\
\hline Differentiating NB & 21 & $13(61.9)$ & \\
\hline GNB & 15 & $13(86.7)$ & \\
\hline \multicolumn{4}{|l|}{ MYCN } \\
\hline Amplified & 16 & $3(18.8)$ & \multirow[t]{2}{*}{0.010} \\
\hline Nonamplified & 56 & $31(55.4)$ & \\
\hline
\end{tabular}


Table 2. Clinicopathologic and biological factors affecting survival rate

Variable

Clinical stage: advanced

(III and IV) vs early (I, II, and IVS)

MYCN: amplified vs nonamplified

GRP75 expression: negative vs positive

Histology: undifferentiated vs differentiated*

Primary tumor site: adrenal vs extra-adrenal

\begin{tabular}{cc} 
Univariate analysis \\
\hline $\begin{array}{c}\text { Relative risk } \\
\text { (95\% confidence interval) }\end{array}$
\end{tabular}

$3.667(1.425-9.488)$

$14.654(3.501-61.337)$

$\begin{array}{lr}6.216(2.971-13.004) & <0.001 \\ 5.369(2.438-11.824) & <0.001 \\ 2.247(1.151-4.385) & 0.018 \\ 1.591(0.807-3.135) & 0.180\end{array}$

\begin{tabular}{cc}
\multicolumn{2}{c}{ Multivariate analysis } \\
\hline $\begin{array}{c}\text { Relative risk } \\
\text { (95\% confidence interval) }\end{array}$ & $\boldsymbol{P}$ \\
\hline $1.143(0.372-3.511)$ & 0.816 \\
$8.551(1.527-47.869)$ & 0.015 \\
& \\
$3.088(1.275-7.478)$ & 0.012 \\
$2.705(1.070-6.838)$ & 0.035 \\
$1.009(0.446-2.285)$ & 0.983 \\
$1.046(0.472-2.317)$ & 0.911
\end{tabular}

*Including differentiating NB and GNB identification of NB tumor biomarkers. Using this approach, we have successfully identified and validated a new prognostic factor GRP75 that correlates with the clinical outcomes of NB patients. Presently, a common strategy for the identification of clinical biomarkers has been to find the differentially expressed proteins in tumor tissues but not in nontumor counterparts by using proteomic analysis for the identification of new biological markers of cancers (14). However, this strategy is not suitable for the study of $\mathrm{NB}$ due to the following two reasons. Firstly, there is usually no nontumor counterpart in a NB surgical specimen for comparison. Secondarily, NB represents a heterogeneous spectrum of tumors ranging from undifferentiated to mature histologies. Accordingly, the protein expression profiles from the tumor tissues could be highly varied from patient to patient, and there is no signature expression profile for a group of tumors with different clinical features. To circumvent these problems, we have resorted to a human NB cell line SH-SY5Y for the proteomic analyses. This human NB cell line established from NB patient's tumor tissue represents a group of relatively
A Patients with undifferentiated NB

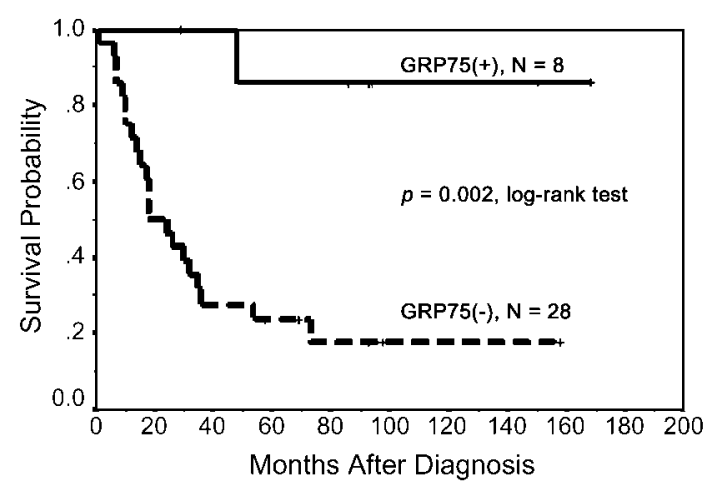

B Patients with differentiated NB

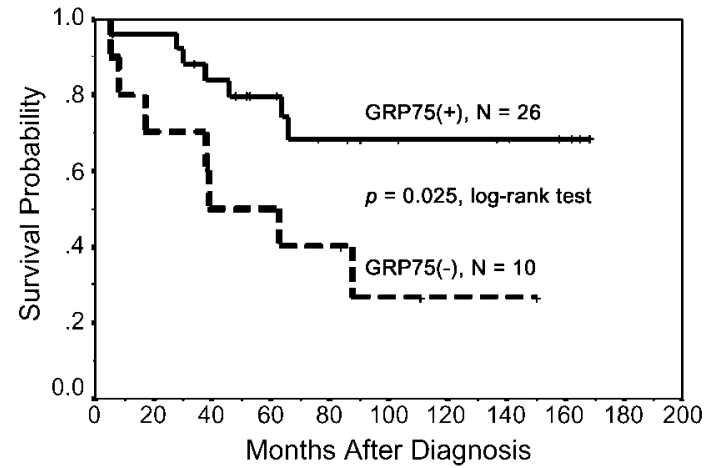

C Patients of advanced disease

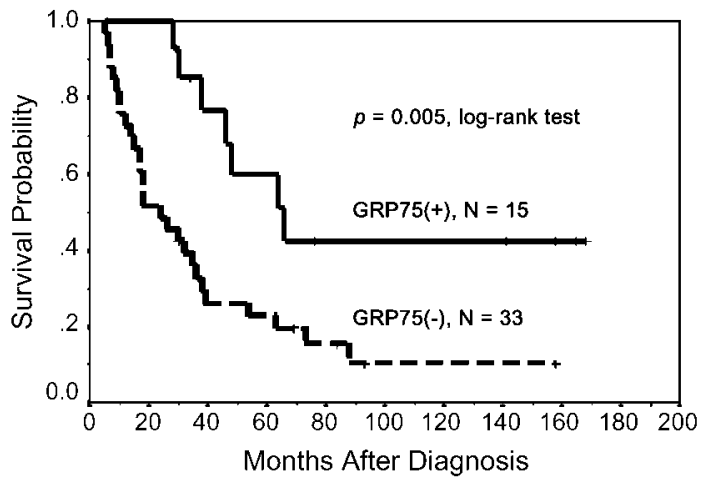

D Patients without MYCN amplification

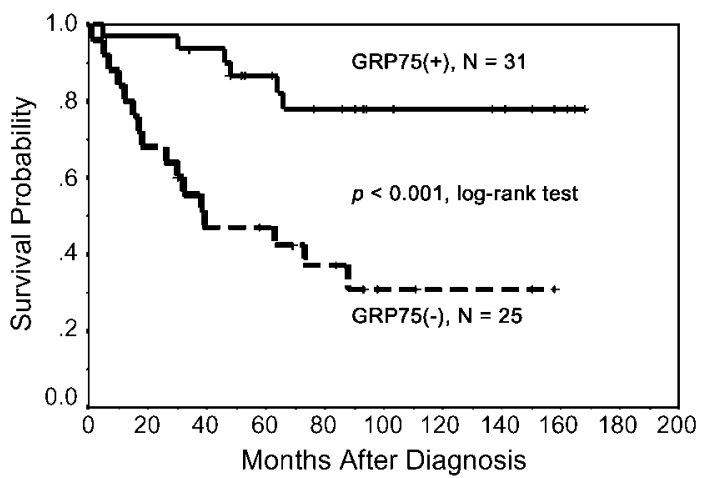

Fig. 4. Kaplan-Meier survival analysis according to the expression of GRP75 in $(A)$ patients with undifferentiated NB, $(B)$ patients with differentiated NB (including differentiating NB and GNB), (C) NB patients of advanced stages (stage III and IV), and $(D)$ NB patients without MYCN amplification. 
homogeneous cells with stable gene expressions recapitulating nicely the in vivo biological characteristics. In addition, these NB cells possess a great potential to differentiate in vitro, which is coupled well with the clinical tumor behavior $(4,6$, 8 ). Therefore, the NB cell line can serve as an ideal model for clinical investigations.

We employ a systematic proteomic approach by using twodimensional DIGE analysis to identify differentially expressed proteins between these proteomes derived from SH-SY5Y cells before and after ATRA-induced differentiation (27). Our screening results in the identification of GRP75 as a novel biomarker associated with the differentiation of NB. The enhanced expression of GRP75 in SH-SY5Y cells after differentiation depicted by two-dimensional DIGE is quantitatively confirmed by Western blotting (two-dimensional and onedimensional) and visualized by immunofluorescence staining (Figs. 1 and 2). Due to the component heterogeneity of the NB tumors, the lack of clear correlation between the histologic grade of differentiation and the protein levels of GRP75 was observed in whole tumor lysates (Fig. 2A; refs. 12, 26). This discrepancy was then resolved by specifically assessing the GRP75 expression in NB cells by immunohistochemical analyses. The results show that the intensity and percentage of GRP75 immunostaining indeed correlates well with the histologic grade of differentiation and the clinical stage of tumors (Fig. 3; Table 1). Most significantly, positive GRP75 immunostaining strongly correlated with the clinical factors of tumors detected by mass screening as well as early clinical stages (Table 1). Interestingly, NB tumors that are either at early clinical stages or detected by mass screening are inclined to spontaneous differentiation or regression (4). Altogether, our results favor that the expression of GRP75 correlates preferentially with the differentiation of $\mathrm{NB}$ cells both in vitro and in vivo

Survival analysis has shown that positive GRP75 expression predicts a favorable prognosis in NB patients independent of age, clinical stage, tumor histology, or MYCN status. In addition, although GRP75 expression correlates well with histologic grade of differentiation, its expression predicts a favorable prognosis in patients with either undifferentiated or differentiated histologies of NB (Fig. 4A and B). These findings suggest that GRP75 could negatively regulate the growth of NB by promoting its differentiation. Furthermore, although clinical stage and MYCN status are two well-recognized most important clinical and biological prognostic factors for $\mathrm{NB}$ $(3,25)$, NB patients with advanced stages (stages III and IV, 48 of 72 patients, 5-year survival rate of $33.7 \%$ ) and absence of MYCN amplification (56 of 72 patients, 5-year survival rate of $68.8 \%$ ) are actually two large group of prognostic heterogeneity. Additional factors are required for further discrimination of these two groups of patients. Although GRP75 expression cannot differentiate the prognostic difference among NB patients with early stages or those with MYCN amplification, our analyses show that positive GRP75 expression predicts a favorable prognosis among NB patients with advanced stages as well as those with tumors carrying normal MYCN copy number (Fig. 4C and D). Thereby, assessing GRP75 expression in NB may provide complementary prognostic information in addition to clinical stage and MYCN status, which in turn may help to determine the most appropriate therapy for the NB patients.
It has been shown that GRP75 is up-regulated in various cancer tissues, cancer cell lines, as well as immortalized cell lines, suggesting that up-regulation of GRP75 may contribute to tumorigenesis (28). However, our present study establishes a novel role of GRP75 in NB by showing that up-regulation of GRP75 is closely correlated with the differentiation of NB cells both in vitro and in vivo. GRP75 is initially known as mortalin due to its presence in the cytosolic fraction of mortal cell phenotype (15). Subsequent studies revealed that normal mortal cells exhibit a pancytoplasmic distribution of GRP75, whereas immortal cells display a perinuclear distribution (29). It has also been shown that nondividing cells express higher levels of GRP75 than dividing cells do (30). In addition, normal brain neurons and ganglion cells express high levels of GRP75 in the cytoplasm $(30,31)$. It is thus plausible that GRP75 could contribute to the nondividing, fully differentiated phenotype of normal neuronal and ganglion cells. Consistent with this notion, our present data show that the expression of GRP75 is evident in the cytoplasm of the mature ganglion cells or differentiating NB cells in the tumor tissue and that differentiated SH-SY5Y cells exhibit a pancytoplasmic and neuritic distribution of GRP75 (Figs. 2 and 3). Whether GRP75 localized at different intracellular compartments can play distinctive cellular functions is not clear. Nevertheless, our data show for the first time that the change in the intracellular distribution of GRP75 coincides with the development of neuronal phenotypes of differentiated NB cells.

A recent study has shown that GRP75 and GRP78, another favorable prognostic marker of NB (11), could bind to receptor for hyaluronan-mediated motility (RHAMM) with an associated down-regulation of RHAMM in Jurkat cells (32). The GRP75/78-RHAMM complex could then bind to the microtubules to stabilize the microtubules in the interphase and prevent the depolymerization of microtubules for the progression of mitosis (32). The essential role of RHAMM in neurite extension has been suggested (33), and the expression of RHAMM has been linked to the progression and metastasis of a variety of cancers (34). Our preliminary data show that SH-SY5Y cells treated with ATRA do exhibit reduced expression of RHAMM, correlating with enhanced neuronal differentiation (data not shown). The possibility thus exists that the pancytoplasmic GRP75 in differentiating NB cells, along with GRP78, may prevent these cells from engaging into mitosis by binding with and down-regulating RHAMM while promoting the neurite formation simultaneously. In addition, although GRP75 can predict the outcome of NB patients independent of other clinicopathologic factors, GRP75 expression is not an independent prognostic factor when GRP78 expression is also placed into the multivariate Cox regression model (data not shown). This finding suggests a possible synergistic correlation between these two heat shock protein 70 family members, GRP75 and GRP78, in affecting NB tumor behavior.

In summary, our study showed that by using a systematic proteomic analysis of NB cells we are able to identify GRP75 as a new prognostic factor of NB. Expression of GRP75 correlates well with the differentiation of NB cells both in vitro and in vivo. Evaluation of GRP75 expression in tumor tissues of $\mathrm{NB}$ may provide complementary prognostic information for further subclassification of these tumors, which in turn may help to 
determine the most appropriate strategy of treatment. Furthermore, because the expression of GRP75 strongly correlates with the differentiation of NB, GRP75 allows itself as a potential target of the treatment of NB. Together, the present data delineate a good example for the employment of proteomic approaches in the identification of potential biomarkers of cancers and recognize the potential of GRP75 as a therapeutic target of NB.

\section{Disclosure of Potential Conflicts of Interest}

No potential conflicts of interest were disclosed.

\section{Acknowledgments}

We thank Dr. Yu-Min Kuo (National Cheng-Kung University) for providing the SH-SY5Y human NB cell line and the Core Facility of the Institute of Cellular and Organismic Biology, Academia Sinica, for technical support.

\section{References}

1. Gurney JG, Severson RK, Davis S, Robison LL. Incidence of cancer in children in the United States. Sex-, race-, and 1-year age-specific rates by histologic type. Cancer 1995; 75:2186-95.

2. Maris JM, Matthay KK. Molecular biology of neuroblastoma. J Clin Oncol 1999;17:2264-79.

3. Brodeur GM. Neuroblastoma: biological insights into a clinical enigma. Nat Rev Cancer 2003;3:203-16.

4. Woods WG, Lemieux B, Tuchman M. Neuroblastoma represents distinct clinical-biologic entities: a review and perspective from the Quebec Neuroblastoma Screening Project. Pediatrics 1992;89:114-8.

5. Hsu WM, Jen YM, Lee $H$, et al. The influence of biologic factors on the surgical decision in advanced neuroblastoma. Ann Surg Oncol 2006;13:238-44.

6. Tonini GP. Neuroblastoma: the result of multistep transformation? Stem Cells 1993;11:276-82.

7. Shimada H, Umehara S, MonobeY, et al. International neuroblastoma pathology classification for prognostic evaluation of patients with peripheral neuroblastic tumors: a report from the Children's Cancer Group. Cancer 2001;92:2451-61.

8. Hoehner JC, Gestblom C, Hedborg F, Sandstedt B, Olsen L, Pahlman S. A developmental model of neuroblastoma: differentiating stroma-poor tumors' progress along an extra-adrenal chromaffin lineage. Lab Invest 1996;75:659-75.

9. Nakagawara A, Arima-Nakagawara M, Scavarda NJ, Azar CG, Cantor AB, Brodeur GM. Association between high levels of expression of the TRK gene and favorable outcome in human neuroblastoma. N Engl J Med 1993:328:847-54.

10. Kitanaka C, Kato K, Ijiri R, et al. Increased Ras expression and caspase-independent neuroblastoma cell death: possible mechanism of spontaneous neuroblastoma regression. J Natl Cancer Inst 2002;94:358-68.

11. Hsu WM, Hsieh FJ, Jeng YM, et al. GRP78 expression correlates with histologic differentiation and favorable prognosis in neuroblastic tumors. Int $\mathrm{J}$ Cancer 2005;113:920-7.

12. Hsu WM, Hsieh FJ, Jeng YM, et al. Calreticulin expression in neuroblastoma-a novel independent prognostic factor. Ann Oncol 2005;16:314-21.

13. Srinivas PR, Verma M, Zhao Y, Srivastava S. Proteo- mics for cancer biomarker discovery. Clin Chem 2002; 48:1160-9.

14. Chatterjee SK, Zetter BR. Cancer biomarkers: knowing the present and predicting the future. Future Oncol 2005;1:37-50

15. Wadhwa R, Kaul SC, IkawaY, Sugimoto Y. Identification of a novel member of mouse hsp70 family. Its association with cellular mortal phenotype. J Biol Chem 1993;268:6615-21.

16. Kaul SC, Taira K, Pereira-Smith OM,Wadhwa R. Mortalin: present and prospective. Exp Gerontol 2002;37: $1157-64$.

17. Wadhwa R, Taira K, Kaul SC. Mortalin: a potential candidate for biotechnology and biomedicine. Histol Histopathol 2002;17:1173-7.

18. Liao YF, Wang BJ, Hsu WM, et al. Unnatural amino acid-substituted (hydroxyethyl) urea peptidomimetics inhibit $\gamma$-secretase and promote the neuronal differentiation of neuroblastoma cells. Mol Pharmacol 2007; 71:588-601.

19. Encinas $M$, Iglesias $M$, Liu $Y$, et al. Sequential treatment of SH-SY5Y cells with retinoic acid and brain-derived neurotrophic factor gives rise to fully differentiated, neurotrophic factor-dependent, human neuron-like cells. J Neurochem 2000;75:991 - 1003.

20. Shimada H, Ambros IM, Dehner LP, Hata J, Joshi VV, Roald B. Terminology and morphologic criteria of neuroblastic tumors: recommendations by the International Neuroblastoma Pathology Committee. Cancer 1999;86:349-63.

21. Shimada H, Ambros IM, Dehner LP, et al. The International Neuroblastoma Pathology Classification (the Shimada system). Cancer 1999;86:364-72.

22. Brodeur GM, Pritchard J, Berthold F, et al. Revisions of the international criteria for neuroblastoma diagnosis, staging, and response to treatment. J Clin Oncol 1993;11:1466- 77 .

23. Tajiri T, Shono K, Fujii Y, et al. Highly sensitive analysis for N-myc amplification in neuroblastoma based on fluorescence in situ hybridization. J Pediatr Surg 1999; 34:1615-9.

24. Kopf I, Hanson C, Delle U,Verbiene I, Weimarck A. A rapid and simplified technique for analysis of archival formalin-fixed, paraffin-embedded tissue by fluores- cence in situ hybridization (FISH). Anticancer Res 1996;16:2533-6.

25. Castleberry RP. Neuroblastoma. Eur J Cancer 1997; 33:1430-7; discussion 7-8.

26. Tanaka $T$, Slamon DJ, Shimoda $H$, et al. Expression of Ha-ras oncogene products in human neuroblastomas and the significant correlation with a patient's prognosis. Cancer Res 1988;48:1030-4.

27. Yan JX, Devenish AT, Wait R, Stone T, Lewis $S$, Fowler S. Fluorescence two-dimensional difference gel electrophoresis and mass spectrometry based proteomic analysis of Escherichia coli. Proteomics 2002;2:1682-98.

28. Wadhwa R, Takano S, Kaur K, et al. Upregulation of mortalin/mthsp70/Grp75 contributes to human carcinogenesis. Int J Cancer 2006;118:2973-80.

29. Wadhwa $R$, Pereira-Smith $O M$, Reddel RR, Sugimoto Y, Mitsui Y, Kaul SC. Correlation between complementation group for immortality and the cellular distribution of mortalin. Exp Cell Res 1995;216: $101-6$.

30. Kaul SC, Matsui M, Takano S, Sugihara T, Mitsui Y, Wadhwa R. Expression analysis of mortalin, a unique member of the Hsp70 family of proteins, in rat tissues. Exp Cell Res 1997;232:56-63.

31. Srokowski T, Pfeifer JD, Li J, Olson LM, Rader JS. Expression and localization of GRP75 in human epithelial tumors and normal tissues. Appl Immunohistochem Mol Morphol 2004;12:132-8.

32. Kuwabara H, Yoneda M, Hayasaki H, Nakamura T, Mori $\mathrm{H}$. Glucose regulated proteins 78 and 75 bind to the receptor for hyaluronan mediated motility in interphase microtubules. Biochem Biophys Res Commun 2006;339:971-6.

33. Nagy Jl, Hacking J, Frankenstein UN, Turley EA. Requirement of the hyaluronan receptor RHAMM in neurite extension and motility as demonstrated in primary neurons and neuronal cell lines. J Neurosci 1995;15: $241-52$.

34. Maxwell CA, Keats JJ, Belch AR, Pilarski LM, Reiman T. Receptor for hyaluronan-mediated motility correlates with centrosome abnormalities in multiple myeloma and maintains mitotic integrity. Cancer Res 2005;65:850-60. 